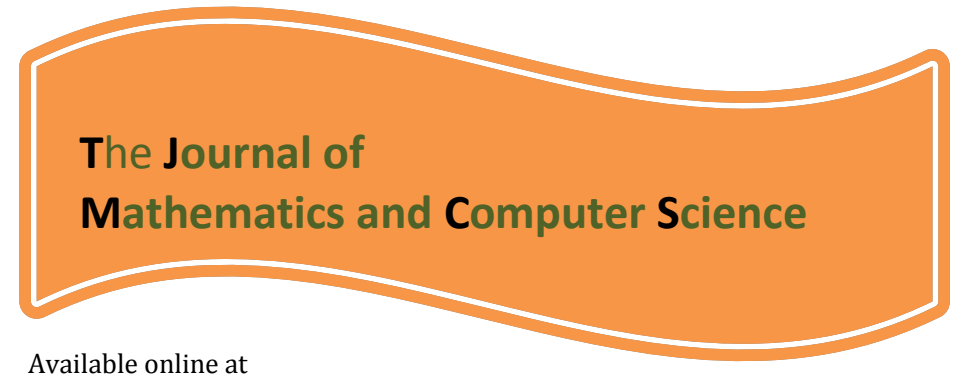

http://www.TIMCS.com

The Journal of Mathematics and Computer Science Vol. 4 No.3 (2012) 487 - 493

\title{
THREE SOLUTIONS FOR A CLASS OF QUASILINEAR DIRICHLET ELLIPTIC SYSTEMS INVOLVING (P, Q)-LAPLACIAN OPERATOR
}

\author{
G.A. AFROUZI' ${ }^{1}$, S. SHAMLO², M. MAHDAVI ${ }^{3}$ \\ ${ }^{1}$ Department of Mathematics, Faculty of Mathematical Sciences, University of Mazandaran, Babolsar, Iran \\ 1afrouzi@umz.ac.ir \\ ${ }^{2}$ s.shamlo847@gmail.com \\ 3mansore.mahdavi@gmail.com
}

Received: February 2012, Revised: May 2012

Online Publication: July 2012

\section{Abstract}

We investigate the existence of three distinct solutions for a class of quasilinearDirichlet ellipticsystems involving the (p,q)-Laplacian operator. Our main tool is a recentthree critical points Theorem of B. Ricceri [On a three critical points theorem, Arch. Math (Basel) 75 (2000) 220-226.

Keywords: Three solutions, Critical points, Dirichlet Systems, Multiplicity result .

\section{Introduction}

In this work, we consider the boundary value system

$$
\left\{\begin{array}{cc}
\Delta_{p}(u)+\lambda f(x, u, v)=a(x)|u|^{p-2} u & \text { in } \Omega \\
\Delta_{q}(v)+\lambda g(x, u, v)=b(x)|v|^{q-2} v & \text { in } \Omega \\
u=v=0 & \text { on } \partial \Omega
\end{array}\right.
$$

Where $\left.\Delta_{s}(u)=\operatorname{div}|\nabla u|^{s-2} \nabla u\right)$ is the s-Laplacian operator, $\Omega \subseteq R^{N}(N \geq 2)$ is a non-empty bounded open set with smooth boundary $\partial \Omega, p, q>N, \lambda>0$ and $f, g: \Omega \times R^{2} \rightarrow R$ is a continuous functions differentiable and the positive weight functions $a(x), b(x) \in C(\bar{\Omega})$.

\footnotetext{
${ }^{1}$-Corresponding author
} 
To be precise, we deal with the existence of an open interval $\Lambda \subseteq[0,+\infty$ [and a positive real number $q$, such that, for $\lambda \in \Lambda$,problem (1.1) admits at least three weak solutions whose norms in $W_{0}^{1, p}(\Omega)$ are less than $q$.

In the literature many papers $[1,2,4]$ discuss quasilinear elliptic systems. For example in [6] the authors studied a class of quasilinear elliptic systems involving the p-Laplacian operator where the right hand side is closely related to the critical Sobolevexponet and they proved the existence of at least one nontrivial solution under suitable assumptions on the nonlinearities. In [7], A. Kristaly using an abstract critical point result of $B$. Ricceriestablished the existence of an interval $\Lambda \subseteq[0,+\infty$ [ such that for each $\lambda \in \Lambda$ the quasilinear elliptic system

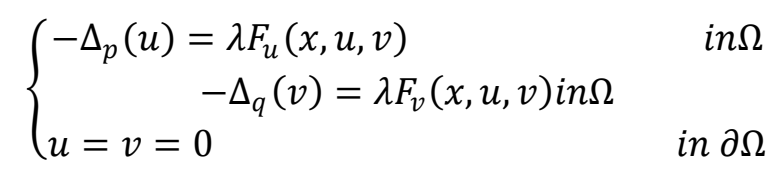

Where $\Omega$ is a strip-like domain and $\lambda>0$ is a parameter, has at least two distinct nontrivial solutions and in [8] Chun-Li and Chun-Lei Tang established the existence of an interval $\Lambda \subseteq[0,+\infty$ [ and a positive real number $\rho$ such that for each $\lambda \in \Lambda$ problem above admits at least three weak solutions whose norms in $W_{0}^{1, p}(\Omega) \times W_{0}^{1, q}(\Omega)$ are less than $\rho$. In [4] authors prove existence of at least three solutions for the problem

$$
\left\{\begin{array}{l}
\Delta_{p}(u)+\lambda f(x, u)=a(x)|u|^{p-2} u \\
u=0
\end{array}\right.
$$

with use of a recent critical points Theorem of B. Ricceri [9]. By a solution (weak) of problem (1.1),we mean any $(u, v) \in X$ such that

$$
\begin{aligned}
& \int_{\Omega}\left(|\nabla u(x)|^{p-2} \nabla u(x) \nabla h_{1}(x)+a(x)|u(x)|^{p-2} u(x) h_{1}(x)\right) d x+ \\
& \int_{\Omega}\left(|\nabla v(x)|^{q-2} \nabla v(x) \nabla h_{2}(x)+b(x)|v(x)|^{q-2} v(x) h_{2}(x)\right) d x+ \\
& \lambda\left(-\int_{\Omega} f(x, u(x), v(x)) h_{1}(x)-g(x, u(x), v(x)) h_{2}(x)\right) d x=0
\end{aligned}
$$

In the sequel, $\mathrm{X}$ will denote the Sobolev space $W_{0}^{1, p}(\Omega) \times W_{0}^{1, q}(\Omega)$ equipped with the norm

We define

$$
\|(u, v)\|=\|u\|+\|v\| \text { Where }\|u\|=\left(\int_{\Omega}|\nabla u(x)|^{p} d x\right)^{1 / p},\|v\|=\left(\int_{\Omega}|\nabla v(x)|^{q} d x\right)^{1 / q}
$$

Put

$$
\|u\|_{1}=\left(\int_{\Omega}|\nabla u(x)|^{p}+a(x)|u(x)|^{p} d x\right)^{1 / p},\|v\|_{2}=\left(\int_{\Omega}|\nabla v(x)|^{q}+b(x)|v(x)|^{q} d x\right)^{1 / q}
$$

$$
k=\max \left\{\sup _{u \in W_{0}^{1, p}(\Omega) \backslash\{0\}} \frac{\max _{x \in \bar{\Omega}}|u(x)|^{p}}{\|u\|^{p}}, \sup _{v \in W_{0}^{1, q}(\Omega) \backslash\{0\}} \frac{\max _{x \in \bar{\Omega}}|v(x)|^{q}}{\|v\|^{q}}\right\}
$$

Since $p, q>N$ one has $k<+\infty$.moreover, from [11] one has 


$$
\sup _{u \in W_{0}^{1, p}(\Omega)\{(0\})} \frac{\max _{x \in \bar{\Omega}}|u(x)|}{\|u\|} \leq \frac{N^{-1 / p}}{\sqrt{\pi}}\left[\Gamma\left(1+\frac{N}{2}\right)\right]^{1 / N}\left(\frac{p-1}{p-N}\right)^{1-1 / p}[m(\Omega)]^{1 / N-1 / p}
$$

And

$$
\sup _{v \in \mathbb{W}_{0}^{1, p}(\Omega)\{0\}} \frac{\max _{x \in \bar{\Omega}}|v(x)|}{\|v\|} \leq \frac{N^{-1 / q}}{\sqrt{\pi}}\left[\Gamma\left(1+\frac{N}{2}\right)\right]^{1 / N}\left(\frac{q-1}{q-N}\right)^{1-1 / q}[m(\Omega)]^{1 / N-1 / q}
$$

Where $m(\Omega)$ is the lebesgue measure of the set $\Omega$, and equality occurs when $\Omega$ is aball. hence, $\operatorname{In} W_{0}^{1, p}(\Omega) \times W_{0}^{1, q}(\Omega)$ the norm $\|(u, v)\|_{1}=\|u\|_{1}+\|v\|_{2}$ is equivalent to the usual one. For all $c>0$ we denote by $K_{1}(c)$ the set

$$
\left\{\left(t_{1}, t_{2}\right) \in R^{2}: \frac{\left|t_{1}\right|^{p}}{p}+\frac{\left|t_{2}\right|^{q}}{q} \leq c\right\}
$$

Put

$$
\begin{gathered}
K\left(x, t_{1}, v(x)\right)=\int_{0}^{t_{1}} f(x, \xi, v(x)) d \xi, E\left(x, u(x), t_{2}\right)=\int_{0}^{t_{2}} g(x, u(x), \eta) d \eta \\
w(x, u(x), v(x))=(K(x, u(x), v(x))+E(x, u(x), v(x))
\end{gathered}
$$

Now, fix $x^{0} \in \Omega$ and pick $r_{1}$, $r_{2}$ with $0<r_{1}<r_{2}$ such that

\section{Main result}

$$
S\left(x^{0}, r_{1}\right) \subset S\left(x^{0}, r_{2}\right) \subseteq \Omega
$$

First we here recall for the readers convenience the three critical points theorem of [9] and proposition 3.1 [10] and proposition 1 [5]:

Theorem 2.1.Let $X$ be a separable and reflexive real banach space; $\phi: X \rightarrow R$ a continuously Gateaux differentiable and sequentially weakly lower semi continuous functional whoseGateaux derivative admits a continuous invers on $X^{*} ; \psi: X \rightarrow R$ a continuously Gateauxdifferentiable functional whose Gateaux derivative is compact. Assume that

$$
\lim _{\|u\| \rightarrow+\infty}(\phi(u)+\lambda \psi(u))=+\infty
$$

forall $\lambda \in[0,+\infty[$, and that there exists a continuous concave function $h:[0,+\infty[\rightarrow R$ such that

$$
\sup _{\lambda \geq 0} \inf _{u \in x}(\phi(u)+\lambda \psi(u)+h(\lambda))<\inf _{u \in x} \sup _{\lambda \geq 0}(\phi(u)+\lambda \psi(u)+h(\lambda))
$$

Then, there exists an open interval $\Lambda \subseteq[0,+\infty[$ and a positive real number $q$ such that, foreach $\lambda \in \Lambda$, the equation

$$
\phi^{\prime}(u)+\lambda \psi^{\prime}(u)=0
$$

has at least three solution in Xwhose norms are less than $q$.

Proposition 2.2. Let $X$ be a non-empty set and $\phi, J$ two real functions on $X$. Assume that there are $r>0$ and such that

$$
\begin{aligned}
& \phi\left(x_{0}\right)=J\left(x_{0}\right)=0, \phi\left(x_{1}\right)>r \\
& \sup _{\left.x \in \phi^{-1}(1-\infty, r]\right)} J(x)<r \frac{J\left(x_{1}\right)}{\phi\left(x_{1}\right)}
\end{aligned}
$$

Then, foreach $\rho$ satisfying 


$$
\sup _{\left.\left.x \in \phi^{-1}(]-\infty, r\right]\right)} J(x)<\rho<r \frac{J\left(x_{1}\right)}{\phi\left(x_{1}\right)}
$$

One has

$$
\sup _{\lambda \geq 0} \inf _{x \in X}(\phi(x)+\lambda(\rho-J(x)))<\inf _{x \in X} \sup _{\lambda \geq 0}(\phi(x)+\lambda(\rho-J(x))) . .
$$

Proposition 2.3.Let $T: X \rightarrow X^{*}$ be the operator defined by

$$
\begin{aligned}
& T(u, v)\left(h_{1}, h_{2}\right)=\int_{\Omega}\left(|\nabla u(x)|^{p-2} \nabla u(x) \nabla h_{1}(x)+a(x)|u(x)|^{p-2} u(x) h_{1}(x)\right) d x \\
& +\int_{\Omega}\left(|\nabla v(x)|^{q-2} \nabla v(x) \nabla h_{2}(x)+b(x)|v(x)|^{q-2} v(x) h_{2}(x)\right) d x
\end{aligned}
$$

For every $(u, v),\left(h_{1}, h_{2}\right) \in X$. Then Tadmits a continuous invers on $X^{*}$.

Now, we state our main result:

Theorem 2.4.Let $f, g: \Omega \times R^{2} \rightarrow R$ be functions such that $f\left(., t_{1}, t_{2}\right), g\left(., t_{1}, t_{2}\right)$ are continuous in $\bar{\Omega}$ for all $\left(t_{1}, t_{2}\right) \in R^{2}, f(x, .,),. g(x, . .$.$) is C^{1}$ in $R^{2}$ and exist two positive constans $\gamma, \beta$ such that $\gamma<p, \beta<q$ and a positive functions $\eta \in L^{1}$ such that

(i) $w(x, u(x), v(x)) \geq 0$

$$
\text { (ii) } \frac{\int_{\Omega} \sup _{\left(t_{1}, t_{2}\right) \in K_{1}(k r)} w\left(x, t_{1}, t_{2}\right) d x}{r}<\frac{\int_{\Omega} w(x, u(x), v(x)) d x}{\frac{\|u(x)\|_{1}^{p}}{p}+\frac{\|v(x)\|_{2}^{q}}{q}}
$$

Where $K_{1}(k r)=\left\{\left(t_{1}, t_{2}\right) \in R^{2} ; \frac{\left|t_{1}\right|^{p}}{p}+\frac{\left|t_{2}\right|^{q}}{q} \leq k r\right\}$, (see (1.3)) and kis given by (1.2) ;

(iii) $w(x, u(x), v(x)) \leq \eta(x)\left(1+\left|t_{1}\right|^{\gamma}+\left|t_{2}\right|^{\beta}\right)$ for almost every $x \in \Omega$ and for all $\left(t_{1}, t_{2}\right) \in R^{2}$, Then, there exists an open interval $\Lambda \subseteq[0,+\infty[$ and a positive real number qsuch that, for each $\lambda \in \Lambda$, problem (1.1) admits at least three solutions in $\mathrm{X}$ whose norms are less than $\mathrm{q}$.

Proof.For $u, v \in X$, we put

$$
\begin{aligned}
\phi(u, v) & =\frac{1}{p}\|u\|_{1}^{p}+\frac{1}{q}\|v\|_{2}^{q}, \\
\psi(u, v) & =-\int_{\Omega} w(x, u(x), v(x)) d x
\end{aligned}
$$

Since p, $\mathrm{q}>\mathrm{N}, \mathrm{X}$ is compactly embedded in $C^{0}(\bar{\Omega}) \times C^{0}(\bar{\Omega})$ and it is well know that $\phi$ and are well defined and continuously Gateaux differentiable functionals whose Gateaux derivatives at the point $(u, v) \in X$ are the functionals $\phi^{\prime}(u, v), \psi^{\prime}(u, v) \in X^{*}$, given by 


$$
\begin{gathered}
\phi^{\prime}(u, v)\left(h_{1}, h_{2}\right)=\int_{\Omega}\left(|\nabla u(x)|^{p-2} \nabla u(x) \nabla h_{1}(x)+a(x)|u(x)|^{p-2} u(x) h_{1}(x)\right) d x \\
+\int_{\Omega}\left(|\nabla v(x)|^{q-2} \nabla v(x) \nabla h_{2}(x)+b(x)|v(x)|^{q-2} v(x) h_{2}(x)\right) d x
\end{gathered}
$$

And

$$
\psi^{\prime}(u, v)\left(h_{1}, h_{2}\right)=-\left(\int_{\Omega} f(x, u(x), v(x)) h_{1}(x) d x+\int_{\Omega} g(x, u(x), v(x)) h_{2}(x) d x\right)
$$

For every $\left(h_{1}, h_{2}\right) \in X$. Hence, the weak solution of (1.1) are exactly the solution of the equation $\phi^{\prime}(u, v)+\lambda \psi^{\prime}(u, v)=0$

respectively, as well as $\psi$ is sequentially weakly upper semicontinuous. We claim that $\psi^{\prime}: X \rightarrow X^{*}$ is a compact operator. Indeed for fixed $(u, v) \in X$, assume $\left(u_{n}, v_{n}\right) \rightarrow(u, v)$ weakly in $X$ as $n \rightarrow+\infty$. Then $\left(u_{n}, v_{n}\right) \rightarrow(u, v)$ strongly in $C(\Omega)$. Since $f(x, .,),. g(x, \ldots)$ is $C^{1}$ in $R^{2}$ for every $x \in \Omega$, so it is continuous in $R^{2}$ for every $x \in \Omega$, and we get that $f\left(x, u_{n}, v_{n}\right) \rightarrow f(x, u, v), g\left(x, u_{n}, v_{n}\right) \rightarrow g(x, u, v)$ strongly as $n \rightarrow+\infty$. By the Lebesguecontrolconvergence theorem, $\psi^{\prime}\left(u_{n}, v_{n}\right) \rightarrow \psi(u, v)$ strongly which means that $\psi^{\prime}$ is strongly continuous, then it is a compact operator. Hence the claim is true. Furthermore, proposition 2.3gives that $\phi^{\prime}$ admits a continuous invers on $X^{*}$ and since $\phi^{\prime}$ is monotone, we obtain that $\phi$ is sequentially weakly lower semicontinuous (see [12, proposition 25.20 ] ) .

Thanks to (iii), for each $\lambda>0$ one has that

$$
\lim _{\|u\| \rightarrow+\infty}(\phi(u, v)+\lambda \psi(u, v))=+\infty
$$

and so one of the assumptions of theorem 2.1 holds. Moreover, since

$$
\sup _{x \in \Omega}|u(x)|^{p} \leq k\|u\|^{p} \text { and } \sup _{x \in \Omega}|v(x)|^{q} \leq k\|v\|^{q}
$$

For each $(u, v) \in X$, we see that

$$
\sup _{x \in \Omega}|u(x)|^{p} \leq k\|u\|_{1}^{p} \text { and } \sup _{x \in \Omega}|v(x)|^{q} \leq k\|v\|_{2}^{q}
$$

For each $(u, v) \in X$, and so

$$
\sup _{x \in \Omega}\left(\frac{|u(x)|^{p}}{p}+\frac{|v(x)|^{q}}{q}\right) \leq k\left(\frac{\|u(x)\|_{1}^{p}}{p}+\frac{\|v(x)\|_{2}^{q}}{q}\right)
$$

For each $(u, v) \in X$, thus we have

$$
\begin{aligned}
\left.\left.\phi^{-1}(]-\infty, r\right]\right) & =\{(u, v) \in X ; \phi(u, v) \leq r\}=\left\{(u, v) \in X ; \frac{\|u\|_{1}^{p}}{p}+\frac{\|v\|_{2}^{q}}{q} \leq r\right\} \\
& \subseteq\left\{(u, v) \in X ; \frac{|u(x)|^{p}}{p}+\frac{|v(x)|^{q}}{q} \leq k r \forall x \in \Omega\right\}
\end{aligned}
$$

and it follows that

$$
\begin{gathered}
\sup _{\left.(u, v) \in \phi^{-1}(1-\infty, r]\right)}(-\psi(u, v))=\sup _{\left.(u, v) \in \phi^{-1}(1-\infty, r]\right)} \int_{\Omega} w(x, u(x), v(x)) d x \\
\leq \int_{\Omega} \sup _{\left(t_{1}, t_{2}\right) \in K_{1}(k r)} w\left(x, t_{1}, t_{2}\right) d x .
\end{gathered}
$$


Therefore, from (ii), we have

$$
\begin{gathered}
\sup _{\left.\left.(u, v) \in \phi^{-1}(]-\infty, r\right]\right)}(-\psi(u, v))=\sup _{\left.(u, v) \in \phi^{-1}(1-\infty, r]\right)} \int_{\Omega} w(x, u(x), v(x)) d x \\
\leq \int_{\Omega} \sup _{\left(t_{1}, t_{2}\right) \in K_{1}(k r)} w\left(x, t_{1}, t_{2}\right) d x . \\
<r \frac{\int_{\Omega} w(x, u(x), v(x)) d x}{\frac{\|u\|_{1}^{p}}{p}+\frac{\|v\|_{2}^{q}}{q}}=r \frac{(-\psi(u, v))}{\phi(u, v)},
\end{gathered}
$$

Fix $\rho$ such that

$$
\sup _{\left.(u, v) \in \phi^{-1}(1-\infty, r]\right)}(-\psi(u, v))<\rho<\frac{(-\psi(u, v))}{\phi(u, v)},
$$

and define $h(\lambda)=\lambda \rho$ for every $\lambda \geq 0$, from proposition 2.2, with $x_{0}=(0,0), x_{1}=(u, v), J=-\psi$, we obtain

$$
\sup _{\lambda \geq 0} \inf _{(u, v) \in X}(\phi(u, v)+\lambda \psi(u, v)+\rho \lambda)<\inf _{(u, v) \in X} \sup _{\lambda \geq 0}(\phi(u, v)+\lambda \psi(u, v)+\rho \lambda) .
$$

Now, our conclusion follows from theorem $2.1 \square$

\section{References}

[1] Afrouzi, G.A., and Heidarkhani, S.,"Existence of three solutions for a class of Dirichletquasilinear elliptic systems involving the $p_{1}, \ldots, p_{n}$-Laplacian", Nonlinear Anal. 70 (2009), 135-143.

[2] Afrouzi,G.A. ,Heidarkhani,S., and O'Regan, D. "Three solutions to a class of Neumann doubly eigenvalue elliptic systems driven by a $p_{1}, \ldots, p_{n}$-Laplacian", Bulletin of the Korean Mathematical Society, to appear.

[3] Afrouzi,G.A.,Heidarkhani,S.,"Existence of three solutions for a Dirichlet boundary value problem involving the p-Laplacian", Nonlinear Anal. 66 (2007), 2281-2288.

[4] Boccardo,L.,Figueiredo,D.,"Some remarks on a system of quasilinear elliptic equations", Nonlinear Differential Equations Appl. 9 (2002), 309-323.

[5] Bonanno, G.,Heidarkhani,S., and O'Regan,D.," Multiple solutions for a class of Dirichletquasilinear elliptic systems driven by a (p,q)-Laplacian operator, Dynamic Systems and Applications, 20 (2011), 89100.

[6] Djellit,A.,Tas,S., "Quasilinear elliptic systems with critical sobolev exponent inR ${ }^{\mathrm{N}}$ ", Nonlinear Anal. 66 (2007) 1485-1497.

[7] Kristaly,A., "Existence of two non-trivial solutions for a class of quasilinear elliptic variational systems on strip-like domain", Proc. Edinb. Math.Soc. 48 (2005), no.2 465-477.

[8] Chun Li, Chun-Lei Tang, "Three solutions for a class of quasilinear elliptic systems involving the (p,q)Laplacian", Nonlinear Anal. 69 (2009) 3322-3329.

[9] Ricceri,B., "On a three critical points theorem", Arch. Math. (Basel),75 (2000), 220-226.

[10] Ricceri,B., "Existence of three solutions for a class of elliptic eigenvalue problem", Math. Comput.Modelling, 32 (2000) 1485-1494 
[11] Talenti, G., "Some inequalities of Sobolev type on two-dimensional spheres, in: W. Walter (Ed.), General Inequalities", vol. 5, in: Internat. Ser. Numer. Math., Birkhauser, Basel, 80 (1987), 401-408.

[12] Zeidler,E., "Nonlinear Functional Analysis and its Applications", vol. II/B, Berlin-Heidelberg-New York, 1990. 